\title{
Processus de fragmentation des structures spatiales du Grand Santiago (Chili)
}

Processes of fragmentation of the spatial structures of Greater Santiago (Chile)

Fragmentierungsprozesse der Raumstrukturen in Groß Santiago (Chile)

\section{Severino Escolano Utrilla et Jorge Ortiz Veliz}

\section{(2) OpenEdition}

Journals

Édition électronique

URL : http://journals.openedition.org/rge/325

DOI : $10.4000 /$ rge.325

ISSN : $2108-6478$

Éditeur

Association des géographes de l'Est

Édition imprimée

Date de publication : 1 juin 2005

Pagination : 195-208

ISSN : 0035-3213

\section{Référence électronique}

Severino Escolano Utrilla et Jorge Ortiz Veliz, "Processus de fragmentation des structures spatiales du Grand Santiago (Chili)», Revue Géographique de l'Est [En ligne], vol. 45 / 3-4 | 2005, mis en ligne le 10 juin 2009, consulté le 08 septembre 2020. URL : http://journals.openedition.org/rge/325 ; DOI : https://doi.org/10.4000/rge.325

Ce document a été généré automatiquement le 8 septembre 2020.

Tous droits réservés 


\section{Processus de fragmentation des structures spatiales du Grand Santiago (Chili) ${ }^{1}$}

Processes of fragmentation of the spatial structures of Greater Santiago (Chile)

Fragmentierungsprozesse der Raumstrukturen in Groß Santiago (Chile)

\section{Severino Escolano Utrilla et Jorge Ortiz Veliz}

\section{NOTE DE L'ÉDITEUR}

Article reçu le 22 août 2002, accepté le 30 janvier 2006

En voyageant on se rend compte que les différences se perdent : chaque ville ressemble peu à peu à toutes les autres, les lieux échangent forme, ordre, distances, une poussière informe envahit les continents. Votre atlas garde les différences intactes : cet ensemble de qualités qui sont comme les lettres du nom. Italo Calvino : Les villes invisibles

\section{Introduction : l'émergence des changements dans le Grand Santiago}

1 Les processus intenses de toutes natures qui agissent depuis plusieurs décennies sur les villes ont entraîné de grands changements, qui sont aussi nets dans la configuration et la physionomie des réseaux urbains qu'en ce qui concerne les fonctions et l'organisation internes de l'espace urbain.

2 Bien que le changement soit la caractéristique vitale la plus permanente des villes, il est évident que dans l'histoire urbaine on peut identifier des périodes de relative stabilité 
alternant avec d'autres caractérisées par de fortes transformations, comme celle que l'on observe depuis les années quatre-vingt à Santiago.

3 Ces processus se manifestent pleinement, et parfois de façon spectaculaire, dans les grandes métropoles qui occupent les nœuds centraux du réseau mondial d'échange de biens, de services et d'information. Cependant, le développement n'est pas linéaire, ni homogène, ni simultané dans toutes les villes d'un pays ou dans toutes les parties d'une ville; au contraire, la structure et les formes présentent des disparités et même des contradictions, entre des villes voisines ou entre des zones de la même ville. Ces contradictions résultent des interactions multiples entre facteurs locaux à différentes échelles.

4 Le Grand Santiago est un paradigme de ces métamorphoses. Durant les 25 dernières années, les autochtones et les visiteurs ont pu constater, parfois avec étonnement, les multiples transformations qui ont modifié la structure et le paysage de Santiago, et ont contribué à augmenter considérablement l'extension de la zone urbanisée. Même une visite rapide de certains secteurs permet de constater les mutations qui se sont produites dans la ville et qui sont d'une telle portée, qu'elles ont changé presque entièrement l'aspect de certains secteurs. L'étalement considérable de la zone urbanisée, la désagrégation du tissu urbain, la polarisation et la ségrégation sociale qui persiste, la prolifération de quartiers fermés (condominiums), la privatisation des infrastructures, des équipements et des espaces publics, et d'autres changements qui se sont produits dans toutes les structures, ont eu de multiples conséquences dans l'ensemble des domaines : sociales, économiques, culturelles, territoriales...

5 Les habitants du Grand Santiago peuvent constater quotidiennement que les distances pour aller au travail, à l'école, faire les courses, ou tout simplement passer un moment de loisir, s'allongent et exigent de plus en plus de temps. On peut supposer que des déplacements que l'on pouvait autrefois faire à pied, nécessitent aujourd'hui le recours aux transports publics ou à la voiture individuelle, ce qui contribue à l'accroissement des embouteillages quotidiens et à l'augmentation de la pollution atmosphérique. Les styles et les conditions de la vie ont changé non seulement pour les individus et les familles, mais aussi pour les institutions, les entreprises et d'autres organismes qui doivent s'adapter à un scénario physique et normatif nouveau.

6 S'il est déjà difficile de démêler les nœuds des relations entre les agents, il est tout aussi ardu de distinguer les forces, les moyens et les effets qui interviennent dans les processus, ainsi que d'évaluer les résultats des changements.

7 Quelques groupes sociaux, et aussi quelques écoles de pensée estiment que le développement urbain récent est un facteur très positif, car il constitue un puissant moteur qui stimule l'économie métropolitaine, tout en se révélant comme un mécanisme efficace d'accumulation de bénéfices et un secteur générateur de revenus et d'emploi.

8 Mais si à ce cadre d'évaluation économico-idéologique on ajoute d'autres dimensions, comme la «durabilité » sociale et environnementale, entendue comme l'amélioration de la qualité et de l'efficacité de l'espace construit, des conditions de vie des citoyens, et de l'équité et de la cohésion sociale, alors l'appréciation que l'on peut porter sur ces évolutions se modifient : les projets développés dans le troisième tiers du $\mathrm{XX}^{\mathrm{e}}$ siècle et au seuil du XXIe ont fait émerger une ville plus complexe mais plus fragmentée dans sa structure sociale et spatiale : en témoignent les nombreux fragments du tissu urbain déconnectés du noyau principal, la persistance de la polarisation sociale et 
l'augmentation de la ségrégation socio-spatiale qui, en outre, se manifeste à de multiples échelles.

9 Le savoir géographique constitue une base scientifique particulièrement adéquate pour s'interroger sur les changements découlant de l'expansion urbaine récente, en particulier ceux qui touchent aux structures spatiales, aux modalités de transformation et à leurs effets de clairs-obscurs.

\section{La complexité des transformations urbaines récentes}

10 Les transformations socio-territoriales récentes du Grand Santiago sont d'une telle importance qu'elles ont affecté toutes les composantes de la vie urbaine, aussi bien les dimensions spatiales et la morphologie urbaine que les structures sociales et fonctionnelles.

11 Dans le processus de recomposition des parties de la ville, un grand nombre de facteurs de nature diverse s'entremêlent dans une trame dense et complexe. On peut citer, entre autres : les caractéristiques physiques du territoire, l'évolution technologique, le mode d'organisation politique, la base économique, le niveau de revenus, le volume et les caractéristiques démographiques et culturelles de la population, les styles de vie...; d'où dérive, en partie, la complexité et la non linéarité des processus.

12 Les changements qui ont eu lieu ou qui surviennent actuellement, éclairent une nouvelle réalité urbaine. On peut associer celles-ci, au moins partiellement, à quelques catégories de villes désignées par des expressions nouvelles qui soulignent les traits considérés comme définissant celles-ci : villes globales (Sassen, 1991), villes informelles (Castells, 1995), villes diffuses (Monclús, 1998) villes archipel (Kunzmann, 2001 ; cité dans Borsdorf, 2004 : 28). Beaucoup de processus et de transformations récentes du Grand Santiago correspondent très bien au phénomène " postsuburbia », tel que le caractérise Borsdorf (2004), en particulier ceux qui sont relatifs aux structures spatiales.

\section{A. Objectifs : la fragmentation des structures spatiales en tant qu'élément du processus de restructuration générale}

Dans ce mouvement de restructuration générale, qualitative et quantitative, il se dégage quelques changements bien visibles constituant des thèmes récurrents de la recherche sur la ville de Santiago. Il s'agit d'une part, de l'augmentation de la polarisation et de la ségrégation sociale et d'autre part, de l'étalement urbain de grande ampleur.

14 Il est évident que ces processus génèrent également des changements dans les autres parties de la ville : dans le centre et le péri-centre on observe un déclin, mais aussi un certain renouvellement (gentrification), des pertes de population dans certaines zones, un accroissement de la densité dans d'autres; le modèle fonctionnel traditionnel monocentrique se désagrège et se réorganise en un autre multipolaire; le paysage urbain semble être dominé par des singularités architecturales qui sont l'expression de la post-modernité.

15 Une des transformations les plus importantes a concerné les structures spatiales du Grand Santiago, qui se sont modifiées très rapidement. Malgré leur évidence, il n'existe 
pas beaucoup d'études consacrées aux modalités, aux causes, aux effets et aux formes de ces changements. Les travaux de Borsdorf (2003) méritent une mention à part : il y a élaboré un modèle $d u$ "développement urbain, politique, social et économique en Amérique Latine depuis l'époque coloniale jusqu'à l'heure actuelle». Dans la phase actuelle, la « fragmentation » est le « principe de structuration spatiale » $(2003: 41)$. On peut aussi citer les travaux de Borsdorf et Hidalgo (2004 y 2005) et ceux de Ortiz et Aravena (2002), Ortiz et Morales (2002) et Ortiz et Escolano (2005), qui analysent les tendances spatiales des divers phénomènes.

Le "principe de fragmentation" se manifeste parfois de façon évidente: dans la structure de l'espace construit, dans le paysage urbain, dans la distribution spatiale de nombreux phénomènes (population, utilisations du sol) et, probablement aussi dans leur organisation verticale. Plus précisément, l'objectif est :

1. d'estimer la «fragmentation» de quelques structures spatiales du Grand Santiago: de l'espace construit et des utilisations du sol.

2. de suggérer quelques hypothèses pour interpréter les résultats obtenus et les tendances observées.

\section{B. Le cadre théorique : vers l'intégration de domaines autonomes d'explication}

17 Pour interpréter les processus de restructuration récentes, plusieurs théories et hypothèses ont été proposées, de portée différente, et à partir de différents points de vue (exploratoires, analytiques...), puisque les multiples disciplines (géographie, sociologie, économie, urbanisme, sciences environnementales) ont apporté leur contribution. Les données et la connaissance ont permis de caractériser leurs modalités d'action, les agents, les phases et les moyens impliqués, ainsi que leur influence dans le développement récent de Santiago.

18 L'un des consensus les plus fermes qui se dégage de ces études est que la "mondialisation» est considérée comme la force motrice qui alimente les changements à travers des mécanismes et des ressources variés, comme on peut le remarquer dans l'excellente synthèse publiée par De Mattos et al. (2004). De plus, d'autres facteurs d'ordre psychologique, culturel, idéologique ou de style de vie ont également favorisé le développement du modèle urbain dispersé et fragmenté du Grand Santiago.

Bien que l'essentiel des processus sociaux qui contribuent à la restructuration soient suffisamment expliqués, nous pensons qu'en ce qui concerne la dimension spatiale, on n'a pas identifié tous les facteurs, ni reconnu toutes les modalités de leur action, ni les formes associées aux interactions, même si leurs effets ont été décrits.

20 Ce n'est pas seulement la multiplicité des approches géographiques (et nongéographiques) sur la ville qui est à l'origine des difficultés à trouver des explications, mais aussi la complexité de l'objet d'étude. La proposition pour surmonter "l'atomisation théorique » ne veut pas dire que l'on adhère à un modèle unique rendant compte de la totalité de l'espace urbain, ce qui serait également inefficace. Il s'agit plutôt de suivre les voies possibles qui permettent d'articuler plusieurs théories partielles pour en former une nouvelle plus puissante ou, comme le propose Hillier (1998: 1), construire des théories sur des «domaines autonomes" où opèrent des 
« lois » et rechercher ensuite la relation entre les domaines (comme entre l'espace et la société) pour déterminerl'interface qui génère une plus grande complexité.

21 Si l'on accepte le fait que la ville est un système complexe (Allen, 1997; Portugalli, 2000 ; Wilson, 2000), il ne semble pas fantaisiste d'explorer les contenus des théories regroupées sous l'étiquette de la «complexité » (Dauphiné, 2003), pour expliquer et modeler des processus comme la croissance et la différenciation, la spécialisation, les discontinuités spatiales et d'autres aussi importants dans la restructuration urbaine en cours.

Il ne s'agit pas ici de retracer l'utilisation des théories de la complexité pour étudier la ville, mais il faut rappeler quelques principes intégrés dans certaines de ces théories :

1. Les formes, les structures spatiales, sont conceptualisées comme des composants actifs des processus en général, et non comme de simples reflets passifs des processus sociaux. Cette idée implique que la relation entre le «social» et le «spatial» est plus complexe qu'une simple dépendance linéaire de cause à effet. Cette idée est particulièrement importante dans la théorie et dans les méthodes de la "syntaxe de l'espace" " (space syntax), qui est une théorie, une méthode et un langage pour décrire l'espace construit (Hillier et Hanson, 1984 : xi); quelques concepts et processus de la «syntaxe de l'espace » sont utilisés dans cet article.

2. L'échelle, la résolution et les unités d'analyse sont des facteurs essentiels pour connaître la relation entre les niveaux d'organisation, l'émergence de nouvelles propriétés, l'organisation verticale et horizontale des phénomènes pour la connaissance géographique de la ville.

Celles-ci, ainsi que d'autres approches contribuent de façon importante à la géographie urbaine, par rapport à d'autres doctrines qui traitent les formes spatiales, et le territoire en général, comme des accessoires ou comme un support inerte, de purs sous-produits des relations sociales; dans d'autres cas, le territoire est simplement ignoré.

\section{Discontinuités dans l'espace urbain construit}

Toutes les activités urbaines, économiques, de la vie sociale et privée, se développent dans deux grandes catégories élémentaires et complémentaires de l'espace construit: l'une est formée par les bâtiments et l'autre par le système d'espaces qui permet la communication entre ceux-ci. La co-disposition spatiale de ces éléments entraîne des configurations ${ }^{3}$ spécifiques dont les propriétés s'associent à de multiples aspects du fonctionnement des villes, comme la mobilité, la distribution d'activités et d'équipements ou la ségrégation résidentielle.

La croissance récente du Grand Santiago, en particulier celui de la périphérie, a suivi des modèles différents de ceux qui existaient auparavant, qui ont augmenté la fragmentation et la discontinuité de la structure de l'espace construit. Le réseau de rues, comme système continu qui canalise le mouvement, reprend et synthétise très bien le type et la magnitude de quelques changements. On peut remarquer entre autres :

1. La baisse de l'accessibilité de l'espace construit, car le réseau de rues est plus souple et moins poreux pour les véhicules et les piétons; de plus les parcours les plus longs et les plus inhospitaliers sont moins attractifs pour être parcourus.

2. La rupture de la linéarité dominante dans le schéma et les voies traditionnelles, qui est remplacé par un autre de type arborescent dans lequel abondent les segments de rue longs, 
avec de nombreux changements angulaires. La linéarité est un attribut fondamental du réseau de rues pour construire, à partir de celle-ci, une image cohérente de l'espace qui facilite son exploration. personnes. configuration complexe, formée par un gradient descendant centre-périphérie, modifié par les émergences de caractère local. Les densités les plus basses (moins de $45 \mathrm{~m} / \mathrm{ha}$ ) forment un anneau extérieur du Grand Santiago qui coïncide avec le développement périphérique récent. Les valeurs les plus élevées sont disposées en alignements isolés dans le tissu urbain construit principalement entre 1950 et 1980, ou au centre de villes traditionnelles comme La Florida et Quilicura. Quelques «défilés" entre les topographies de cimes reflètent, directement, la présence de voies de transports modernes, qui peuvent difficilement s'appeler «rues» dans leur acception traditionnelle : autoroutes, voies à deux chaussées séparées (Panamericana, del Sol), de grandes rocades ou des voies rapides. Cette répartition met en évidence que les grandes voies urbaines, qui appartiennent essentiellement au modèle d'occupation du sol, sont des éléments physiques importants dans le processus de fragmentation spatiale.

Figure 1 : Densité de rues du Grand Santiago (mètres/hectare).

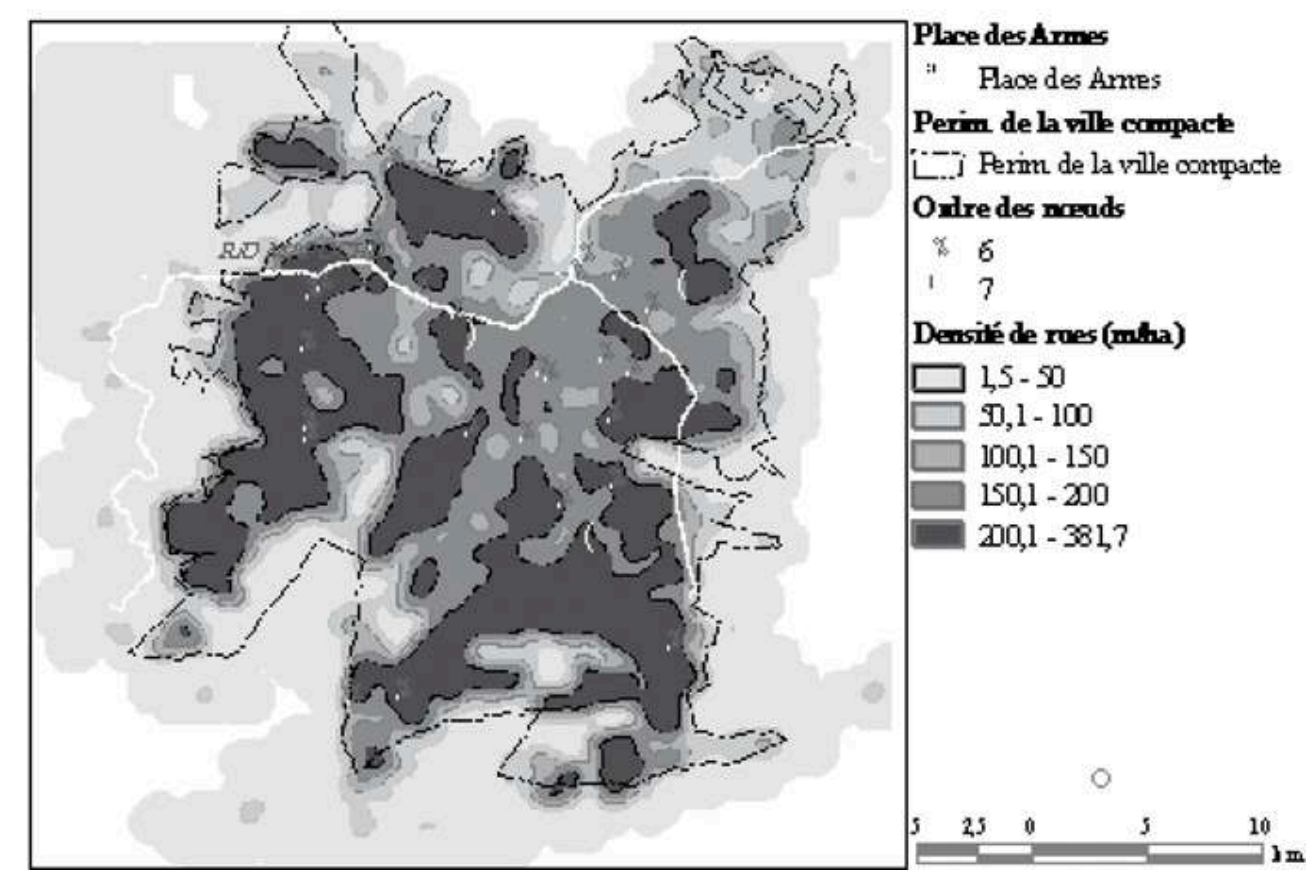

La carte de la figure 2 représente la diversité de nœuds qui peuvent se trouver dans un rayon de 500 mètres depuis n'importe quel lieu. On différencie à nouveau la périphérie récente, pour sa diversité inférieure, par rapport au reste du réseau, où l'on peut trouver au moins un ou plus de nœuds quaternaires à moins de 500 mètres de n'importe quel point; dans la périphérie la proportion de nœuds de troisième ordre (où trois segments s'entrecroisent) est plus élevée que dans le reste de la ville, où s'unit sa linéarité inférieure. La plus grande diversité, naturellement, se trouve autour des 
nœuds de cinq, six ou sept, qui se distribuent de façon plus ou moins régulière dans l'espace construit.

Figure 2 : Diversité de nœuds du réseau de rues du Grand Santiago. 2002.

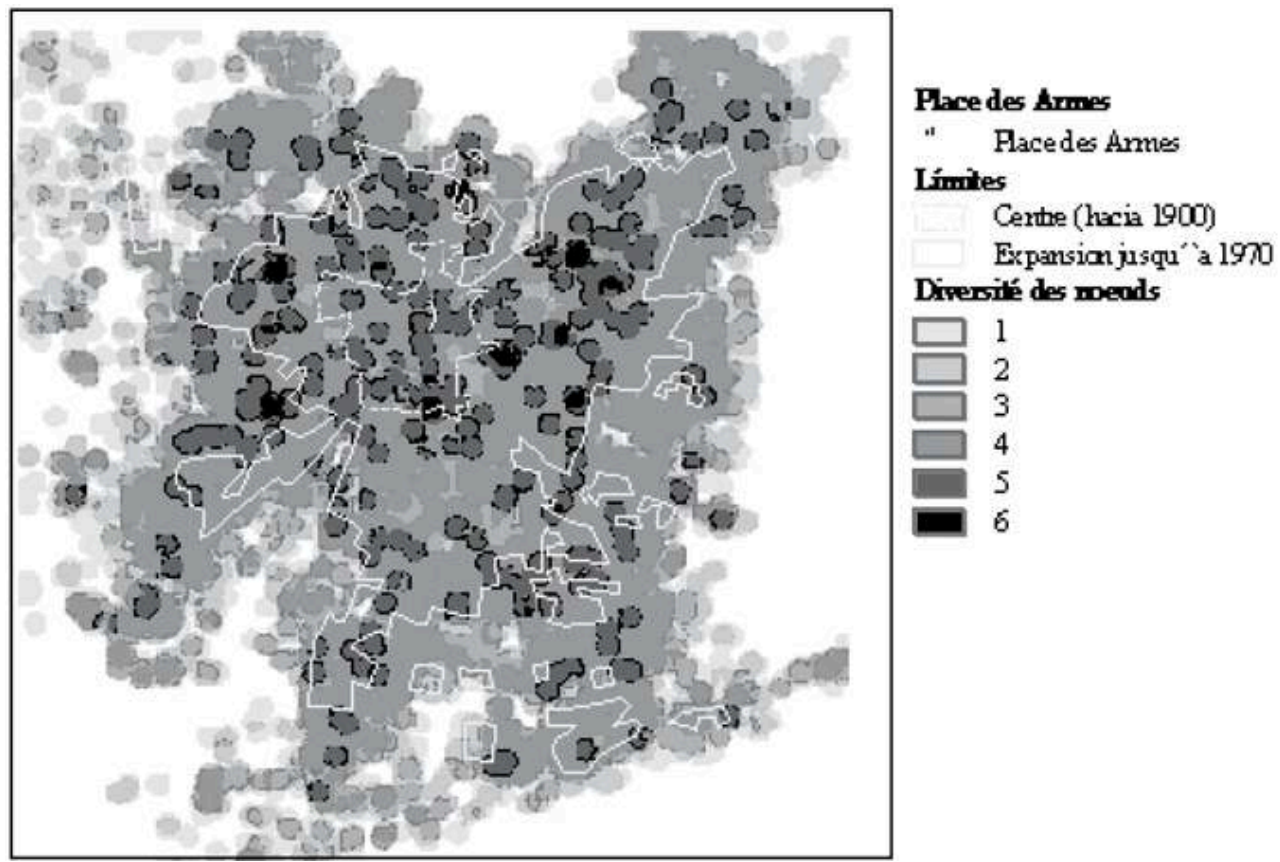

29 Ces cartes montrent que l'accessibilité locale est assez acceptable dans une bonne partie de l'espace urbain construit jusqu'en 1980 (et pour une bonne partie de la population), bien que leur distribution spatiale soit organisée en taches, interrompues par des bandes de faible accessibilité. Sur la périphérie, l'accessibilité locale est assez faible.

\section{A. Hypothèse sur le développement et la conformation de la structure du réseau d'espaces publics du Grand Santiago}

30 Le réseau d'espaces publics du Grand Santiago forme un système complexe, dont le développement et l'organisation se sont produits à partir de la diffusion et de la coalescence du tissu local, en suivant des principes relativement simples: la prolongation d'axes principaux existant déjà qui facilitent la connexion à longue distance, auxquels sont unis des segments de niveau hiérarchique inférieur par lesquels sont conduits les flux locaux.

31 A mesure qu'augmente l'espace urbain, la hiérarchie de chaque rue et segment se réorganise en fonction de sa position géométrique et topologique et d'autres caractéristiques physiques, généralement en relation avec les précédentes (longueur, largeur, etc.). En réalité, toute la structure de l'espace construit obéit à ce même processus fractal, car les bâtiments ne sont que des îlots entourés d'un espace continu de mouvement.

Pour vérifier cette hypothèse, on a comparé deux échantillons du réseau de rues : l'un correspond à une portion de tissu de type « traditionnel » et l'autre au « moderne » très répandu dans la périphérie récente (tableau 1, figure 3). D’autre part, pour tout le 
réseau on a mesuré la relation entre le périmètre et la surface des espaces délimités par les rues.

Tableau 1 : Processus d'expansion du réseau de rues : modèles « traditionnel » et modèle de la " nouvelle périphérie »

\begin{tabular}{|c|c|c|c|c|c|c|}
\hline \multirow[t]{2}{*}{ Ordre des nœuds } & \multicolumn{3}{|c|}{ Modèle «traditionnel » } & \multicolumn{3}{|c|}{ Modèle "nouvelle périphérie " } \\
\hline & $\begin{array}{l}\text { Diamètre } \\
250 \mathrm{~m}\end{array}$ & $\begin{array}{l}\text { Diamètre } \\
500 \mathrm{~m}\end{array}$ & $\begin{array}{l}\text { Diamètre } \\
1000 \mathrm{~m}\end{array}$ & $\begin{array}{l}\text { Diamètre } \\
250 \mathrm{~m}\end{array}$ & $\begin{array}{l}\text { Diamètre } \\
500 \mathrm{~m}\end{array}$ & $\begin{array}{l}\text { Diamètre } \\
1000 \mathrm{~m}\end{array}$ \\
\hline 1 & & & 3 & & 4 & 12 \\
\hline 2 & & & 5 & 2 & 4 & 13 \\
\hline 3 & 20 & 69 & 270 & 3 & 11 & 36 \\
\hline 4 & 6 & 22 & 61 & & 2 & 5 \\
\hline Nombre de nœuds & 26 & 91 & 339 & 5 & 21 & 66 \\
\hline Arêtes & 52 & 168 & 572 & 9 & 34 & 95 \\
\hline $\begin{array}{l}\text { Longueur totale } \\
\text { d'arêtes }(\mathrm{m})\end{array}$ & 1901 & 7481 & 28872 & 694 & 2670 & 8865 \\
\hline $\begin{array}{l}\text { Longueur moyenne } \\
\text { (m) }\end{array}$ & 37 & 45 & 50 & 77 & 78 & 93 \\
\hline Densité (m rue/ha) & 96,84 & 95,26 & 91,90 & 35,35 & 33,99 & 28,21 \\
\hline Surface (ha) & 19,63 & 78,53 & 314,15 & 19,63 & 78,53 & 314,15 \\
\hline Index & & & & & & \\
\hline M & 27 & 78 & 234 & 5 & 14 & 30 \\
\hline $\begin{array}{l}\text { Nombre maximum } \\
\text { d'arcs }\end{array}$ & 47 & 177 & 673 & 5 & 37 & 127 \\
\hline Alfa $(\alpha)$ & 0,57 & 0,44 & 0,35 & 1,00 & 0,38 & 0,24 \\
\hline Bêta $(\beta)$ & 2,00 & 1,85 & 1,69 & 1,80 & 1,62 & 1,44 \\
\hline 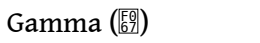 & 0,72 & 0,63 & 0,57 & 1,00 & 0,60 & 0,49 \\
\hline
\end{tabular}

n : nombre de nœuds ; 1 : nombre d'arêtes (links) ; $\mathrm{c}:$ nombre de sub-graphes $=1$

$\mu=I-n+c$; les réseaux denses ont une valeur élevée qui indique que les nœuds sont bien connectés Nombre maximum d'arcs pour connecter tous les nœuds directement $=\mathrm{n}(\mathrm{n}-1) / 2$

$a=(I-n+c) / 2 n-5 ;$ domaine : $0<=a<=1$; est une version standardisée de $\mu$

$\beta=\mid / n$; domaine : $0<=\beta<=(3 n-6) / n$; dans les réseaux denses $\beta$ est élevé et aussi $\mu$ $\gamma=1 /(3 n-6)$; domaine : $0<=\gamma<=1$, est une version standardisée de $\beta$ 
Figure 3 : Échantillons du réseau de rues de type «traditionnel » et de la « nouvelle périphérie ».

\begin{tabular}{|c|c|c|}
\hline Dimensions & Modèle « nouvelle périphérie » & Modèle « traditionnel \# \\
\hline $\begin{array}{l}\text { Rayon des cercles : } 250 \mathrm{~m} \text {; } \\
500 \mathrm{~m} \text {; } 11000 \text {. } \\
\text { Supefficie : } 19,63 \text { ha ; } \\
78,54 \text { ha } ; 314,15 \text { ha } \\
\text { Les symboles ponctuels } \\
\text { représentent les nœuds de } \\
\text { différent ordre. }\end{array}$ & & \\
\hline
\end{tabular}

La première configuration montre comment sa croissance a lieu à travers le remplissage (ou extension) des ressources de l'espace avec des lignes orthogonales qui génèrent des nœuds quaternaires, qui sont les plus fréquents. La distribution statistique des nœuds selon la surface s'ajuste avec une grande précision à une fonction linéaire dont l'équation est $\mathrm{y}=0,0004 \mathrm{x}+6,33\left(\mathrm{r}^{2}=0,99\right)$. Le principe suivi est identique dans le tissu de la périphérie récente, mais maintenant les intersections ne sont pas octogonales (et pas seulement pour des raisons de topographie) et, même si les nœuds de quatrième ordre sont aussi les plus nombreux, les ternaires sont davantage représentés que dans le cas précédent, la relation entre le nombre de nœuds et la surface est également linéaire mais la pente est très inférieure selon l'équation $\mathrm{y}=$ $05 \mathrm{x}-8 \mathrm{E}+2,83\left(\mathrm{r}^{2}=0,99\right)($ figure 4$)$.

Figure 4 : Relation de la superficie, nœuds, arcs et leur longueur. Echantillons du centre et de la périphérie.

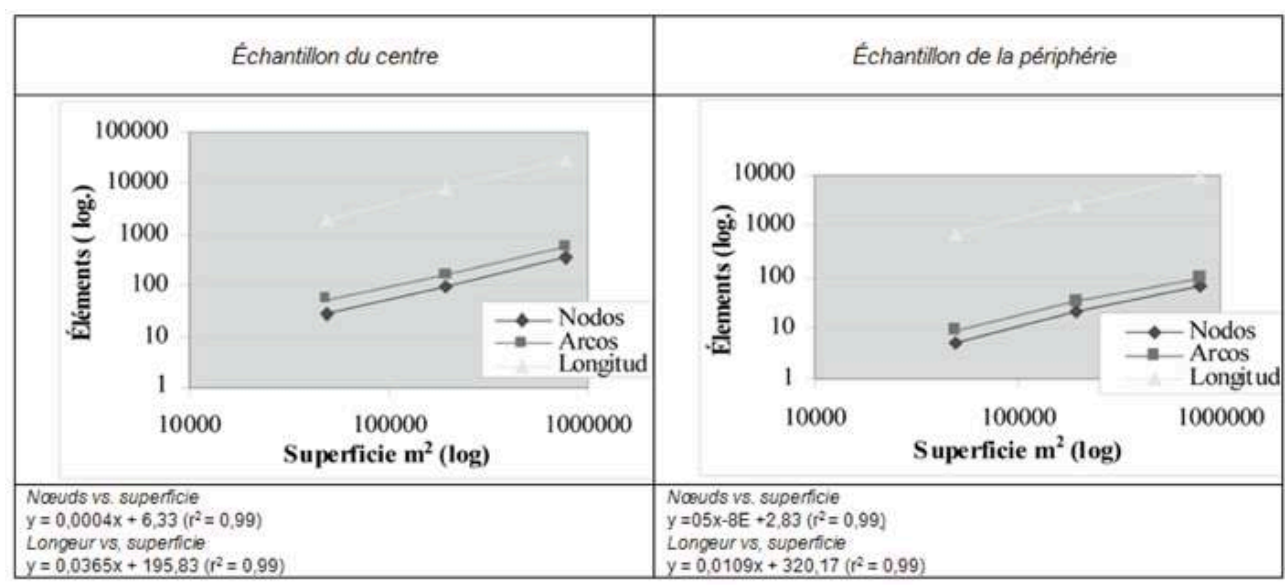

La densité de mètres d'axe de rue en relation avec la surface se trouve autour de $95 \mathrm{~m} /$ ha dans l'exemple du centre, et ce qui est fondamental, elle varie à peine avec l'échelle. Par contre, dans l'exemple de la périphérie cette magnitude est non seulement inférieure, environ $30 \mathrm{~m} / \mathrm{ha}$, mais elle baisse aussi avec l'augmentation de la surface. Cette différence de densité, ajoutée à la forme réticulaire dans le premier cas et arborescente dans le deuxième, entraîne une structure qui produit des distributions locales de l'accessibilité et des configurations spatiales très différentes. Naturellement, ces qualités se transmettent jusqu'au système général. 
En effet, dans le premier cas, il n'existe que peu de différences dans l'accessibilité locale, car presque toutes les routes de parcours minimum ont la même longueur; seulement en changeant d'échelle, les lieux acquièrent une centralité et une meilleure accessibilité, principalement en fonction de leur position géométrique centrale. Par contre, dans les structures de la périphérie, les lieux apparaissent déjà hiérarchisés à une échelle locale, et la différenciation augmente en changeant d'échelle, car il existe des nœuds distributeurs qui débouchent sur d'autres secondaires jusqu'à arriver à des rues sans issue qui laissent la place aux bâtiments.

Le processus de composition de la hiérarchie des nœuds montre aussi des différences avec le changement d'échelle, entre le réseau «traditionnel » et le "modèle de la périphérie ». Dans le premier cas, l'augmentation de la surface est accompagnée de l'apparition de nœuds de différent ordre, jusqu'à arriver à une taille dont la distribution est semblable à celle de toute la ville; cette taille " critique » pourrait se situer entre 300 et 400 ha. Cependant, dans la périphérie, la formation de la hiérarchie de nœuds ne montre pas une corrélation semblable avec l'échelle : la structure semble formée déjà avec environ 100 ha et la composition élémentaire (20 ha) est bien différente du premier type (figure 5).

Figure 5 : Relation entre le nombre et l'ordre des nœuds dans le réseau de rues « traditionnel » et dans celui de la « nouvelle périphérie».

\section{IMAGE MANQUANTE}

Une autre manière indirecte d'estimer la différence de structure des parties du tissu urbain et sa tendance à constituer une maille dense et régulière, consiste à comparer le périmètre et la racine carrée de la surface des différents « îlots » délimités par les rues. Sur la figure 6, on représente cette relation pour le réseau du centre, pour l'espace construit jusqu'à 1970 et pour l'espace développé entre 1970 et 2002. La forme de la relation est identique dans les trois cas, ainsi que l'association, mais les pentes varient de façon significative. Au centre, les zones continues construites sont relativement petites (elles ne dépassent pas les $5 \mathrm{~km}$ de périmètre ni les 5 ha) et assez carrées; dans l'espace développé jusqu'à 1970, la surface inter-route est plus grande et un peu plus irrégulière (le périmètre peut atteindre les $10 \mathrm{~km}$ et la surface $200 \mathrm{ha}$ ); enfin, dans la périphérie récente, ces superficies atteignent de plus grandes tailles et sont irrégulières ( $25 \mathrm{~km}$ de périmètre et $800 \mathrm{ha}$ ). Un coup d'œil sur une carte détaillée ou une image aérienne du Grand Santiago montre clairement la déformation progressive de la maille des rues depuis le centre jusqu’à la périphérie. 
Figure 6 : Relation entre le périmètre et la racine carrée de la superficie des « îlots » dans les différentes étapes de formation du Grand Santiago. 1900, 1970 et 2002.

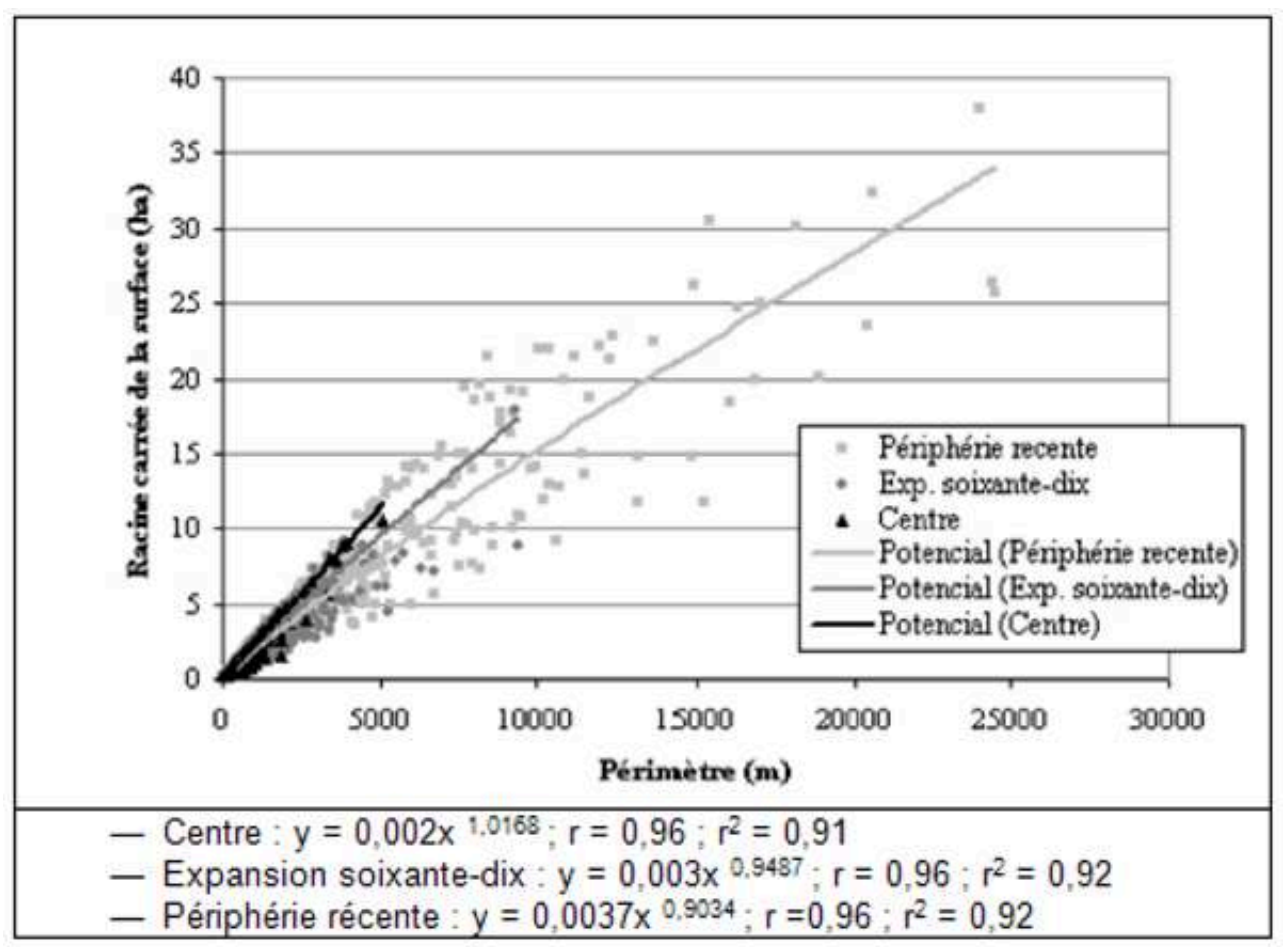
éléments de création de l'espace urbain se diffusent par la structure considérée à d'autres échelles; on admet en outre que l'un des processus dominants de l'organisation urbaine s'opère du bas vers le haut. sur une échelle, pâtés de maison sur l'autre...) et à la manière dont les groupes deviennent des unités à d'autres niveaux supérieurs.

Il semble que, dans une bonne partie du tissu du centre, les bâtiments, ordonnés en pâtés de maisons fermés disposés sur un réseau, sont à l'origine de la structure à n'importe quel niveau considéré.

Cependant, dans d'autres parties, plus étendues sur la périphérie, le processus est plus complexe, car des éléments propres interviennent à plusieurs échelles. Ainsi, le périmètre s'impose (en général d'une longueur considérable), régulier ou irrégulier, défini par une raison (clôture d'une copropriété, adaptation à un chemin préalable ou aux pentes d'un versant) et simultanément, dans cette superficie (non distributive) les unités élémentaires se construisent (maisons) en suivant un modèle, régulier ou irrégulier, mais indépendant de l'espace qui le contient. Dans ce cas, la forme de l'unité supérieure n'émerge pas de la forme des unités élémentaires, comme cela se passe dans le tissu «traditionnel».

Les configurations spatiales décrites sont différentes, tant par leurs dimensions métriques que dans les dimensions topologiques. S'il est évident que la relation de distance et longueur entre les éléments a augmenté dans le nouveau tissu de la périphérie, la perméabilité a également diminué et les espaces publics aptes pour l'«exploration " et pour la maintenance et le développement des relations sociales (espaces assemblés) $)^{5}$ ont disparu. La profondeur topologique ${ }^{6}$ a augmenté d'un ou deux 
niveaux; donc la perméabilité a diminué, ce qui constitue un mécanisme direct qui augmente la ségrégation sociale (figure 7).

Figure 7 : Exemples de configurations spatiales sur tissu traditionnel et de la périphérie selon leur profondeur topologique.

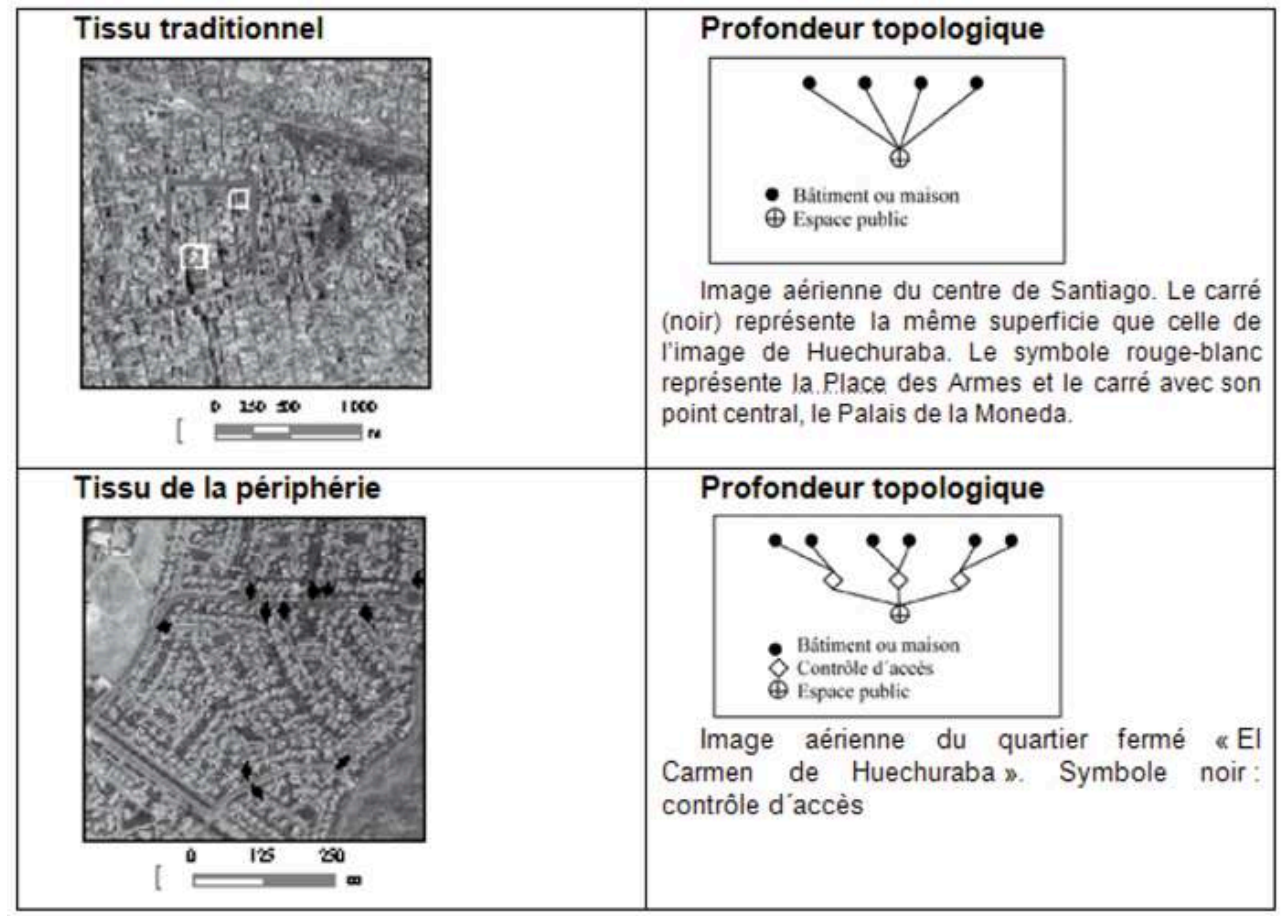

\section{Quelques implications de l'augmentation de la fragmentation de l'espace urbain construit}

La structure spatiale d'une bonne partie de l'espace urbain développé récemment, en particulier sur la périphérie, est caractérisé par :

- L'apparition de nombreux segments de l'espace urbanisé de tailles variées, proches ou relativement éloignés du noyau principal.

- La naissance de toutes sortes de discontinuités sur le tissu urbain de la nouvelle périphérie : plus grandes distances entre les bâtiments, plus grande taille des pâtés de maison d'espace privé entre le réseau des voies publiques, ruptures dans le paysage, autant dans ses composantes visuelles que dans son organisation...

- L'émergence d'une configuration spatiale moins perméable, moins compacte et moins linéaire que celle qui existait déjà. Un système spatial avec ces propriétés exerce une certaine influence dans le fonctionnement général de la ville ; on suggèrera provisoirement quelques-uns de ces effets.

En premier lieu, l'intégration ${ }^{7}$ de l'espace urbain, entendue comme l'accessibilité générale, a diminué. Le mouvement à longue distance est plus coûteux, en raison de la moindre fréquence de longs axes rectilignes dans la périphérie, ou à la noncontinuation d'autres qui existaient auparavant. Pour atteindre quelques secteurs il est 
nécessaire de réaliser de multiples détours et d'utiliser plusieurs rues. Les rues les plus intégrées correspondent aux grands axes urbains, certains développés en sens est-ouest (Alameda) et d'autres unissent des quartiers du sud et le centre urbain (Av. José Carrera, Concha y Toro, Santa Rosa); les rues les plus polarisées sont plus abondantes sur la périphérie.

D'autre part, l'articulation globale-locale est déficiente dans une bonne partie de la périphérie. Le centre urbain est le secteur le mieux relié au système général, c'est-àdire que depuis n'importe quel point il est relativement facile d'arriver à un axe routier principal. Par contre, dans certaines zones de la périphérie, cette organisation se produit à travers plusieurs segments intermédiaires, ce qui augmente leur niveau d'isolement.

Une autre caractéristique importante de la configuration spatiale en cours postsuburbia - est la diminution de l'intelligibilité. Cette propriété décrit un autre aspect de l'interaction globale-locale, et exprime la relation entre l'espace concret de mouvement (ce que l'on voit) et l'image de la structure générale (ce que l'on connaît). Cette notion reprend de riches contenus de l'utilisation et de l'expérience de l'espace, qui peuvent se synthétiser dans l'idée d'« espaces exploratoires » (Read et Budiarto, 2003 : 13.18).

49 Dans plusieurs parties de la périphérie, surtout là où abondent les quartiers fermés par de grandes enceintes, les systèmes de déplacement à longue distance sont en général séparés des systèmes de déplacement local, ce qui entraîne des configurations spatiales peu propices, et même rédhibitoires, pour la naissance du « sens de lieu » et ne favorise pas l'intégration sociale. Bien qu'il s'agisse de zones qui ont, ou peuvent avoir, un degré d'accessibilité acceptable, à travers des ceintures, des voies rapides et des autoroutes urbaines radiales, elles manquent de "perméabilitété »: la configuration spatiale même est non seulement confuse, mais elle est aussi semée d'obstacles qui rendent le mouvement difficile. En effet, les individus doivent passer et parfois choisir entre une densité élevée d'embranchements d'autoroutes, parkings, rampes, couloirs, tours, zones surveillées, ... et toutes sortes de systèmes d'ingénierie et d'architecture installés pour surveiller et diriger le mouvement à travers des niveaux multiples jusqu'à un scénario planifié, public ou semi-public. Ces zones ont seulement quelques points de fixation qui forment un réseau très lâche, physiquement et mentalement habitable en même temps.

\section{A. Désagrégation de la compacité et diminution de l'hétérogénéité du modèle d'utilisation du sol}

50 La structure et la distribution de l'espace occupé pour le développement des activités, c'est à dire des utilisations du sol, représentent une des expressions les plus originales de la complexité des villes. Les « utilisations » sont des attributs des objets physiques, y compris les maisons, qui se manifestent comme des lieux d'origine et de destination des mouvements urbains, espaces de communication ou équipements relatifs à la vie urbaine. Leur localisation, type et dimensions sont des informations importantes pour savoir comment fonctionnent les villes.

51 Les discontinuités mentionnées auparavant dans le tissu urbain construit (réseau de rues lâche et non linéaire, d'abondantes enceintes clôturées, unités très grandes) ainsi que la création de gigantesques centres de commerce et de services, méga-conceptions de 
Borsdorf et Hidalgo (2005), entraînent des impacts dans le modèle d'utilisation du sol, qui évolue vers la désagrégation du schéma monocentrique traditionnel et où apparaissent de grandes zones homogènes et mono-fonctionnelles consacrées presque exclusivement à l'activité résidentielle.

Le développement spectaculaire des moyens de transport permet la séparation spatiale d'activités - utilisations - qui étaient auparavant en contiguïté. Ainsi, de nouvelles zones spécialisées de commerce et services (comme les chaines de commerces et de services relatifs à l'automobile situés le long des axes d'entrée et de sortie des villes) naissent ou se créent hors des centres urbains traditionnels, dans les nœuds centraux des périphéries urbaines (centralisations inverses) autour d'infrastructures d'échelle inédite comme les mall, shopping, et autres regroupements de commerces et services. On observe en même temps le déclin de certains secteurs du centre traditionnel.

En effet, le processus est assez dynamique dans le Grand Santiago. Quelques activités commerciales et de service, et aussi des équipements, ont été déplacés du centre en périphérie, ou bien ont été créés directement ici. Mises à part les formes traditionnelles, l'originalité de ce processus a été l'apparition de concentrations modernes de commerces et de services qui stimulent la formation d'un modèle fonctionnel poly-nucléaire. La localisation de ces agglomérations est justifiée par la bonne accessibilité en automobile. C'est la raison pour laquelle elles se trouvent proches des carrefours principaux, à l'intersection des rocades et des grandes voies radiales.

Malgré cela, les activités très spécialisées et de grande valeur symbolique restent ou se créent dans le centre traditionnel ou dans le «nouveau centre» de Providence. D'un autre côté, ces nœuds attirent aussi vers eux d'autres utilisations du sol parmi lesquelles on trouve la fonction résidentielle.

\section{La mesure de l'hétérogénéité}

55 Pour l'étude de l'organisation spatiale des utilisations du sol nous utiliserons, principalement, les informations concernant la localisation de l'intensité et de la combinaison des utilisations du sol, des mesures réalisées à partir des indicateurs de densité. Les données primaires correspondent aux mètres carrés de dix-huit catégories ${ }^{8}$ d'utilisation, mesurés dans les 1327 zones du Grand Santiago, bien que les données valables touchent 1104 zones.

La multifonction et l'hétérogénéité ainsi que la densité sont des concepts relatifs à l'échelle, et leur variation spatiale dépend des unités de mesure. Une voie habituelle pour examiner sa distribution spatiale est d'atténuer les valeurs par un procédé de filtrage (kernel) (Batty et al, 2003 : 4) qui gradue les changements brusques créés par la prise de données en unités arbitraires discrètes (zones dans ce cas). Les paramètres utilisés ici sont: un filtre circulaire d'un kilomètre de rayon ( 314 ha) avec des pondérations qui suivent une distribution normale; les unités résultantes sont des pixels de 100 mètres de côté. Ces paramètres ont été adoptés après plusieurs essais, et sont ceux qui reflètent le mieux les données de départ.

La diversité a été assimilée à la complexité contenue dans la composition d'utilisations de chaque zone et a été mesurée à partir des probabilités structurelles des types d'utilisation de chaque zone ; l'information moyenne de l'état du système d'utilisations - entropie - a été estimée au moyen de l'équation de Shannon et Weaver : 
$\mathrm{H}=-\Sigma \mathrm{p}_{\mathrm{i}} \log _{2} \mathrm{p}_{\mathrm{i}} \quad$ Où : $\mathrm{i}=1,2,3 \ldots \mathrm{n}$;

$\mathrm{p}_{\mathrm{i}}$ : probabilité qu'un événement i se produise ;

$\Sigma \mathrm{p}_{\mathrm{i}=} 1$

Le résultat s'exprime en bits/individu.

Cette mesure a été assez utilisée dans des analyses écologiques et dans l'évaluation de toutes sortes de complexités; donc ses avantages et ses limites sont bien connues. Cependant, la quantité absolue de diversité dépend, dans une certaine mesure, de la taille de l'unité, donc pour comparer des unités de différentes tailles il faut normaliser les valeurs absolues. Le procédé le plus commun consiste à les adapter à la valeur maximale d'entropie ; l'indicateur résultant est la diversité relative ou densité-diversité (Batty et al. 2003 : 3) et se définit comme suit :

\section{L'intensité d'occupation du sol et sa distribution spatiale}

En général, l'organisation spatiale de l'intensité d'utilisation du sol montre un gradient descendant depuis le « district central des affaires » (CBD), qui coïncide avec le centre historique et son milieu, vers la périphérie. Cependant, cette tendance générale est modifiée localement par une frange de noyaux périphériques assez consolidés.

Le « centre Providence » mérite une mention spéciale puisqu'il est localisé au nord-est de la ville, et se trouve immédiatement après le précédent; tous les deux sont connectés dans l'espace par un couloir avec une densité d'utilisations assez élevée (figure 8).

Figure 8 : Densité d'utilisation totale du sol dans le Grand Santiago. 2002.

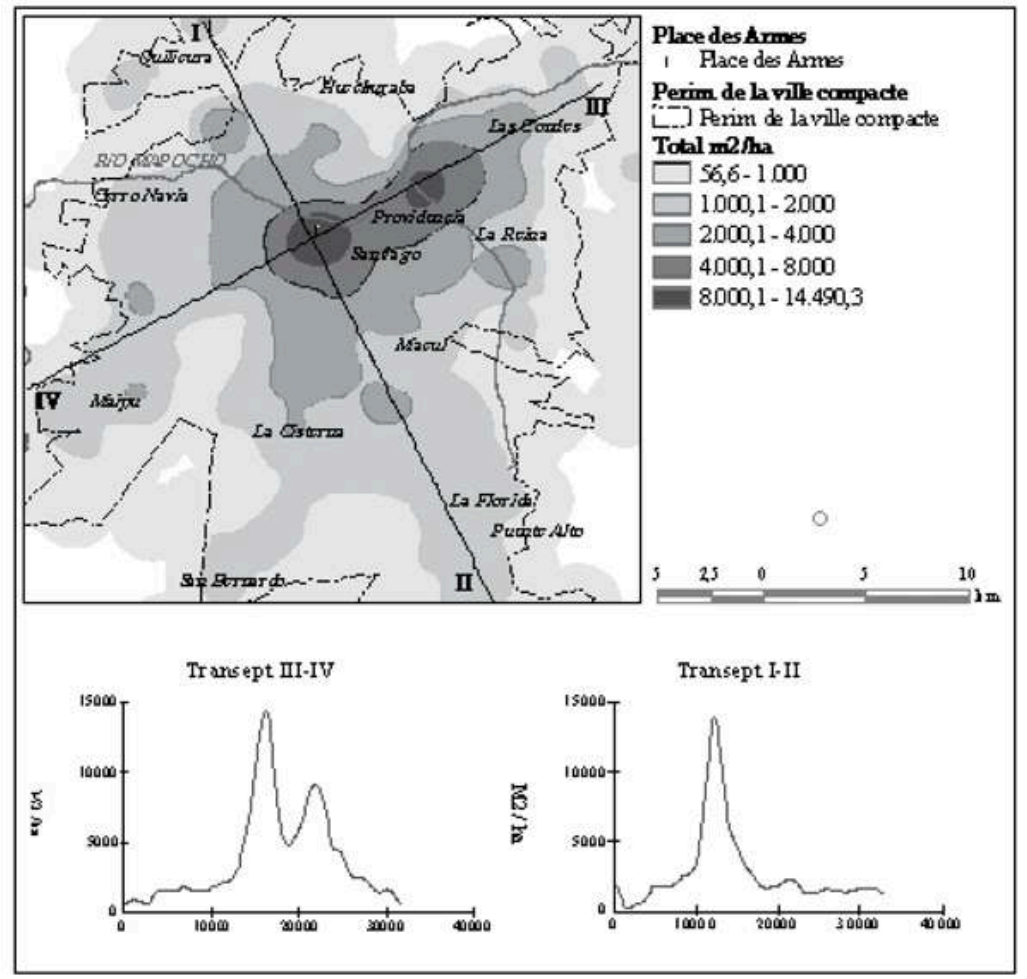


La distribution de valeurs de densité selon la distance au centre (mesure à la Place des Armes) s'ajuste à une distribution logarithmique négative, avec une valeur $r$ de Pearson $r=-0,53\left(r^{2}=0,3\right)$, significatif avec un niveau de probabilité de $95 \%$. C'est à dire que l'intensité d'occupation du sol retombe rapidement à proximité du centre, et à partir d'une distance de plus ou moins cinq kilomètres, elle diminue légèrement (figure 9).

Figure 9 : Relation entre la densité d'utilisation du sol et la distance au centre urbain dans le Grand Santiago.

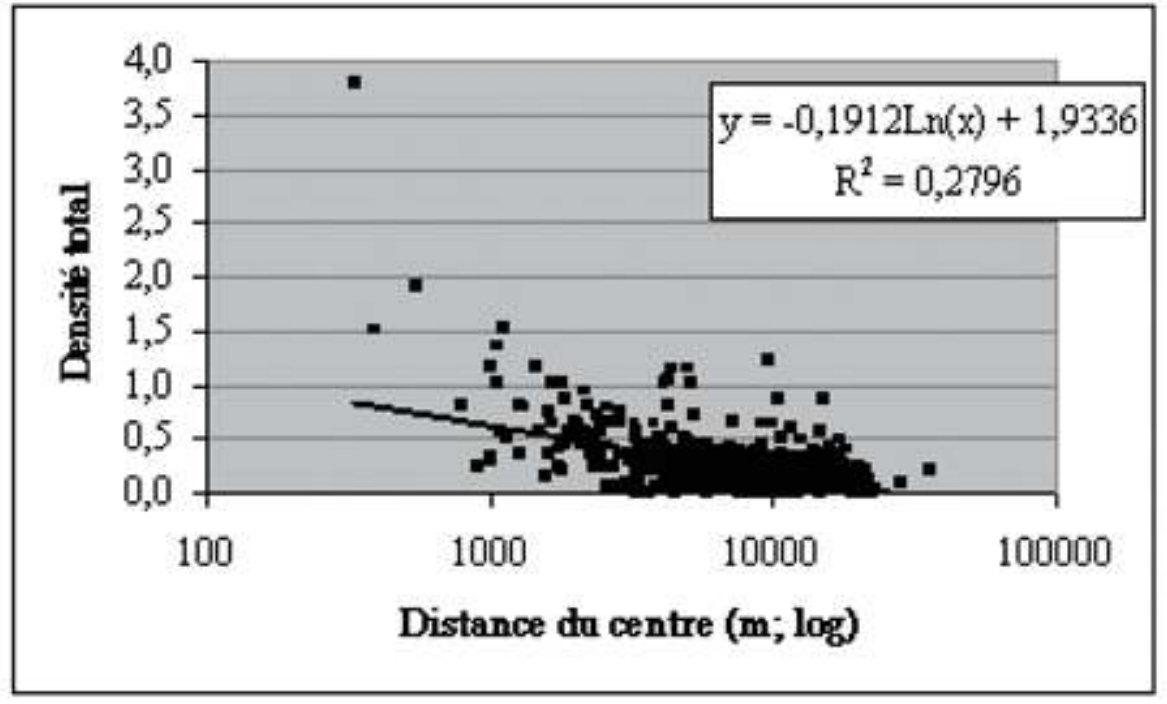

Les résidus de la régression précédente se constatent parfaitement sur la carte de la figure 8 et sur les transects, où des secteurs se profilent, comme le coin Nord-Ouest et quelques îlots à Huechuraba, Quilicura, Peñalolén et d'autres communes, qui sont audessus du rapport moyen, tandis que d'autres zones, comme la frange la plus extérieure, se trouvent au-dessous de celui-ci.

Les figures indiquent que l'organisation des densités d'occupation du sol est gouvernée par la distance au centre, mais aussi que d'autres forces interviennent comme l'accessibilité, la distribution de la population (et de ses caractéristiques démographiques, économiques et culturelles) qui impliquent des modèles indépendants, dispersés ou en noyaux.

66 La distribution spatiale des valeurs totales d'utilisation du sol est composée par des « signatures » différentes correspondant à chaque type de fonction, dont la structure spatiale obéit à des processus propres. L'examen de chaque catégorie d'utilisation révèle des patrons spatiaux différents, conformés par l'accessibilité à la population (équipements en général), par des dépendances fonctionnelles (industrie et stockage ; hôtels, services et bureaux) ou par des facteurs multiples (prix du sol, centralisation, etc.).

\section{La fragmentation de la diversité des utilisations du sol}

67 Comme on l'a indiqué, la diversité est un indicateur de la complexité de la structure d'un système, mesurée dans ce cas à partir des utilisations du sol. Les valeurs peuvent s'interpréter en termes de multi-fonctionnalités et de mélange d'utilisations, de carence ou de richesse d'interactions sociales et, en définitive, comme modèles de 
zones complexes et hétérogènes ou d'autres homogènes et mono-fonctionnelles respectivement associées à la ville traditionnelle et compacte et à la ville dispersée.

La diversité $\mathrm{H}$ calculée par zones atteint une valeur moyenne de 1,20 bit/zone ; il s'agit d'une valeur très basse, surtout comparé avec celles des écosystèmes naturels (le maximal mesuré est $\mathrm{H}=5,3$ ). Sachant que la valeur absolue dépend de la taille des unités spatiales, et comme on ne dispose pas de la même mesure pour d'autres années, nous ne pouvons pas connaître la tendance de la ville bien que, certainement, elle sera ascendante en raison de l'intégration de nouvelles utilisations dans quelques zones. La valeur maximale est de 3,146, et elle est localisée dans une zone de la commune de Santiago, la deuxième et la troisième correspondent à des zones de la commune de Providencia et la quatrième et la cinquième de nouveau à des zones de la commune de Santiago (figure 10).

Figura 10 : Diversité absolue et relative des utilisations du sol dans le Grand Santiago. 2002.

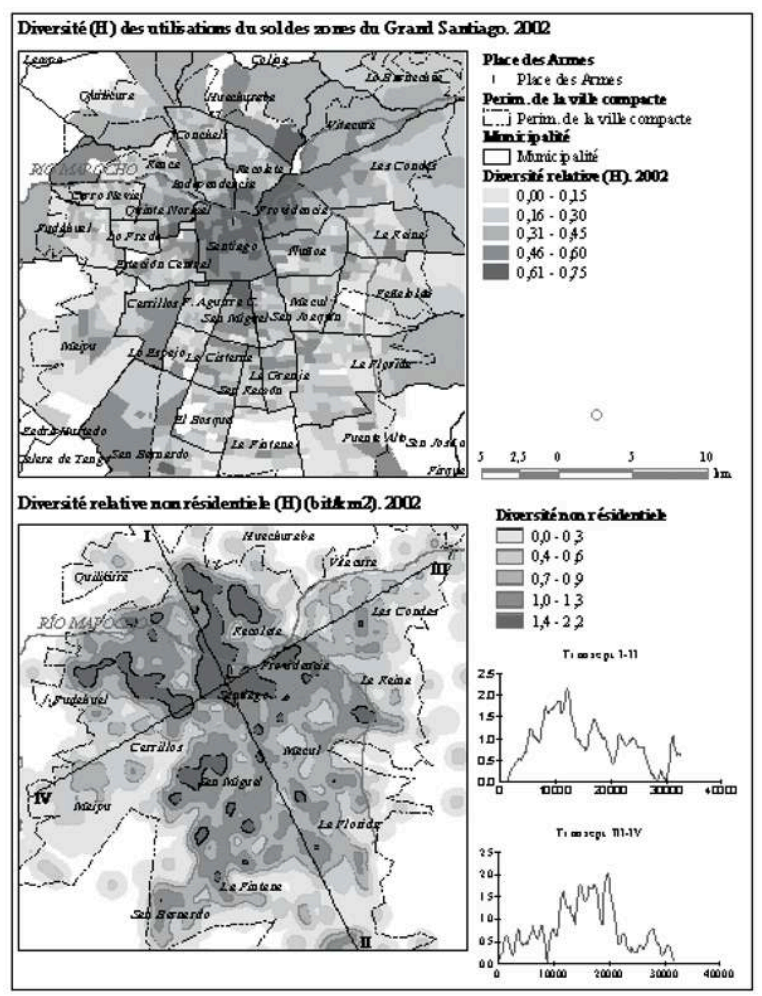

D'autre part, si cette mesure se calcule pour toutes les utilisations du sol, sa distribution compose un modèle assez complexe. Les valeurs les plus élevées, supérieures à 0,5 , sont localisées sur: a) un noyau central assez étendu, approximativement cruciforme, un des bras va de la commune d'Estación Central au sud-ouest jusqu'à Vitacura, Lo Barnechea et Las Condes au nord-est, et l'autre depuis la commune d'Independencia, au nord, jusqu'à celle de San Joaquín et San Miguel au sud ; b) une série de zones périphériques situées sur les bords de la ville compacte dans les communes de Huechuraba, Quilicura, Pudahuel, San Bernardo, Puente Alto, La Florida et La Reina y, c) des zones dispersées interstitielles parmi les secteurs précédents. Les valeurs les plus basses se regroupent dans l'espace en grandes zones qui occupe le péricentre et la périphérie. 
70 Par contre, si l'intensité-densité sont utilisées pour des usages non résidentiels9 (carte de la figure 10), alors le modèle se simplifie considérablement. Les valeurs les plus élevées (supérieures à 1,4 ) se regroupent en un certain nombre de noyaux, séparés entre eux par des espaces plus vastes et plus plats. La tache la plus importante, de forme irrégulière et allongée, se déploie depuis la commune de Santiago et Independencia et Conchalí. D'autres secteurs moins vastes et aussi irréguliers se développent par Estación Central-Lo Prado-Cerro Navia; à Providencia; à Recoleta; à P.A. Cerdá-San Miguel ; à San Ramón ; à San Bernardo ; à La Florida ; à Peñalolén ; et à d'autres foyers plus petits. Autour de ces zones l'intensité diminue. Ce modèle montre déjà sa structure en pièces et la grande extension occupée par des zones homogènes (valeurs faibles) dans la périphérie.

71 La distribution spatiale de la diversité met en évidence la prolongation du noyau central par l'axe nord-ouest et, dans une moindre mesure, vers le sud, ainsi que l'apparition dans la périphérie de nouveaux noyaux de centralisation dispersés avec un niveau élevé de mélange d'utilisations du sol, séparées par des grandes surfaces de sol mono-fonctionnel-résidentiel. Cette configuration est un exemple de plus du processus de formation d'un modèle fonctionnel polycentrique et fragmenté.

\section{B. La mobilité quotidienne : évidence de l'apparition du processus postsuburbia?}

La désagrégation des structures spatiales du tissu urbain et la lente consolidation d'un modèle fonctionnel mono-polycentrique (c'est à dire composé d'un noyau principal (le centre traditionnel et, peut-être Providencia) et de plusieurs noyaux secondaires, dispersés dans la périphérie), ont contribué à modifier les modèles spatiaux de la mobilité quotidienne.

73 Les changements les plus remarquables par rapport au modèle précédent de mouvement peuvent se résumer par :

- La réduction du poids relatif - et peut-être absolu - des flux centre-périphérie.

- L'intensification de courants, ou l'apparition de nouveaux, de type circulaire, c'est-à-dire périphérie-périphérie.

- L'augmentation de la complexité du système quotidien de mobilité.

À partir des données du Recensement de la Population de 2002 (INE) on a construit la carte de la figure 11 qui représente les déplacements entre les communes du Grand Santiago pour des raisons de «travail et d'études ». Pour faciliter la visualisation on a seulement cartographié les flux supérieurs à 10000 personnes. 
Figure 11 : Flux inter-communaux pour travail et études dans le Grand Santiago. 2002.

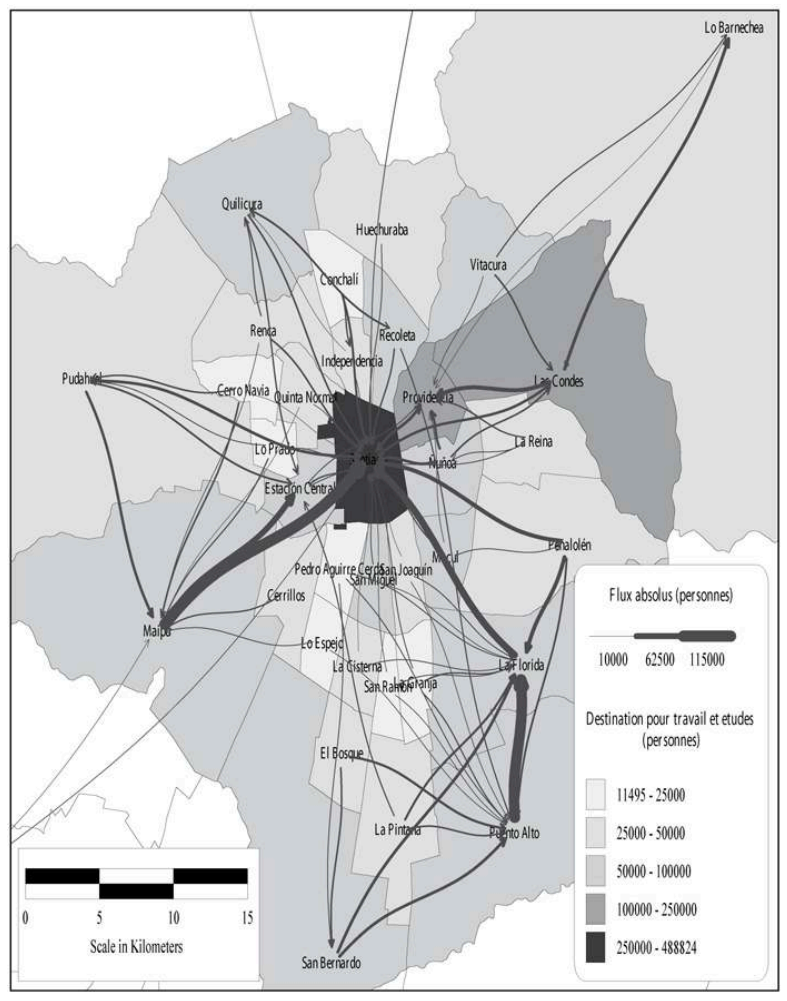

75 La mobilité réelle reprise sur la carte montre le domaine fonctionnel du centre, avec l'apparition de noyaux d'une certaine importance dans la périphérie qui confirment la fragmentation des structures spatiales et la désagrégation du modèle fonctionnel monocentrique; ces tendances seraient aussi vérifiées si l'on introduisait les déplacements pour les achats et les services. Le modèle fonctionnel d'utilisation du sol et celui de la mobilité ressemblent ainsi considérablement au schéma que propose Borsdorf $(2004: 10)$ pour représenter les phénomènes de postsuburbia.

\section{Conclusion}

Le Grand Santiago et une bonne partie de la Région Métropolitaine sont modifiés par des processus généraux de restructuration qui touchent tous les domaines de la vie urbaine : sociale, culturelle, économique, urbanistique, territoriale...

Le détonateur et le moteur principal des changements ont été les activités qui ont défini la «mondialisation », développées à partir de l'ouverture de l'économie et de la société chiliennes (tout au moins d'une partie) vers l'espace économique mondial depuis la deuxième moitié des années 70. La vitesse et l'effervescence des transformations sont propres aux phases initiales des processus, et très dépendantes de l'extérieur, même si la grande taille économique et urbanistique atteinte par le Grand Santiago contribue aussi à son auto-impulsion.

Toutes les parties impliquées dans ce mouvement composent une trame très complexe et difficile à éclaircir en raison de la grande variabilité du nombre, de la nature et des dimensions des agents qui interviennent, des moyens utilisés et des territoires et des groupes sociaux concernés. Pour cela, les conséquences ne sont pas homogènes ni simultanées partout ni dans tous les groupes sociaux, bien qu'ils soient très proches 
dans l'espace : le comportement ressemble plus à celui d'un fluide ou à une matière très élastique qu'à celui d'un solide.

Une des métamorphoses les plus visibles et les plus spectaculaires du Grand Santiago, est celle que manifestent les structures spatiales, entendues comme les modifications de la configuration de l'espace construit, des modèles d'utilisation du sol, de densité de population... Quelle que soit la méthode scientifique employée, et même la simple observation, elles montrent l'énorme expansion de la périphérie récente, l'immensité de ses formes fragmentées et fermées, le gigantisme des interventions - méga projets pour les résidences, les commerces et les services; mais elles révèlent aussi de grandes variations dans le centre traditionnel, dans les utilisations du sol et du bâti. Ces changements et d'autres encore modifient rapidement la physionomie du Grand Santiago et pire encore, son âme.

Face à la bifurcation prise par les structures spatiales, on peut se poser des questions au sujet des caractéristiques et propriétés émergentes des nouveaux modèles spatiaux, entendus comme une partie de l'organisation socio-spatiale générale du Grand Santiago.

81 La réalisation de cet objectif repose sur le postulat qu'il existe un cordon ombilical entre l'ordre social et l'ordre spatial, de telle manière que les structures spatiales introduisent des principes et des formes de l'organisation sociale et en même temps, elles renforcent ou orientent un ordre et un comportement sociaux déterminés.

Les théories de la complexité appliquées à l'explication de la croissance urbaine, en particulier la syntaxe de l'espace, soutiennent que l'ordre de l'espace construit se génère en un processus du bas vers le haut qui est en plus diachronique (et c'est pour cela qu'il dure) et qui s'appréhende à travers la synchronisation cognitive propre des capacités rationnelles des agents urbains. D'autre part, la théorie de l'auto-organisation critique peut apporter de nouvelles voies d'approche aux contenus étudiés ici.

À partir des analyses menées dans ce travail, on peut dégager les points suivants :

1. La texture granulaire de l'espace construit peut s'expliquer comme un phénomène d'émergence à partir de l'utilisation d'unités d'intervention plus grandes, sans qu'il soit nécessaire d'avoir recours à des arguments de conception et de planification centralisés. Cette idée coïncide avec les appréciations de De Mattos (2004: 11) sur la manière isolée et partielle («fédération de communes») de concevoir et d'exécuter le projet, en particulier les infrastructures.

2. Les changements dans le modèle du réseau d'espaces publics dans plusieurs secteurs de la périphérie sont aussi des manifestations émergentes, produites par la grande taille et la forme irrégulière des unités de développement. Les propriétés de ce modèle arborescent conditionnent les utilisations, l'image et l'expérience de ces espaces.

3. Les utilisations du sol façonnent un schéma spatial cohérent avec la localisation de la population et avec les propriétés de l'espace construit et son accessibilité. Le modèle fonctionnel compact et monocentrique se recompose en un autre mono-polycentrique.

84 Ces faits empiriques peuvent s'interpréter, peut-être, comme des évidences de l'intervention de principes de territorialité dans l'organisation spatiale récente du Grand Santiago. Cela veut dire que les groupes sociaux, autant ceux qui ont tendance à s'intégrer dans l'espace que ceux qui ne le sont pas, se réunissent dans des espaces déterminés et tendent à s'exclure. 

renforcer le local, restreint les rencontres, et détermine clairement les frontières et les barrières physiques et psychologiques. Naturellement, leur capacité de survie et de maintenance requièrent une taille minimale, plus grande que celle d'un tissu social ouvert et divers.

86

Grand Santiago est une entité urbaine complexe, différenciée aussi bien dans son aspect social que spatial, dont l'expansion est gouvernée par une logique qui conduit à la ségrégation, à la formation d'une ville composée de fragments homogènes juxtaposés. C'est probablement une forme normalisée d'évolution des grandes villes.

\section{BIBLIOGRAPHIE}

ALLEN P.M. (1997). - Cities and Regions as Self-Organizing Systems: Models of Complexity, Gordon and Breach, Amsterdam.

ASHER F. (1995). - Metapolis ou l'avenir des villes, Odile Jacob, París.

BATTY M., Besussi E., MAAT K., JAAP J. (2003). — « Representing Multifintional cities : Density and Diversity in Sapce », Working Papers, Centre for Advanced Spatial Analysis (CASA), University College, Londres, http://www.casa.ucl.ac.uk

BORSDORF A. (2003). - « Como modelar el desarrollo y la dinámica de la ciudad latinoamericana », Eure, v.29, no $86: 37-49$.

BORSDORF A. (2004). - « On the way to post-suburbia? Changing structures in the outskirts of European cities ». En : Borsdorf, A. y Zembri, P. (eds). European Cities Structures. Insights on Outskirts, COST Action, METL/PUCA, Paris, 6-30.

BORSDORF A. \& HIDALGo R. (2004). - « Formas tempranas de exclusión residencial y el modelo de la ciudad cerrada en América Latina. El caso de Santiago », Norte Grande, 32, 21-37.

BORSDORF A. \& HIDALGO R. (2005). - « Los mega-diseños residenciales vallados en las periferias de las metrópolis latinoamericanas y el advenimiento de un nuevo concepto de ciudad. Alcances en base al caso de Santiago de Chile ", VII Coloquio Internacional de Geocrítica : Los agentes urbanos y las políticas, Santiago de Chile, 24-27 de mayo de 2005, Instituto de Geografía, Pontificia Universidad Católica. www.ub.es/geocrit/7-col.htm

BUREL F. \& BAUNDRY J. (2002). - Ecología del paisaje. Conceptos, métodos y aplicaciones, Mundi Prensa, Madrid.

CASTELLS M. (1995). - La ciudad informacional. Tecnologías de la información, reestructuración económica y el proceso urbano regional, Alianza, Madrid (edición original en 1989).

DAUPHINE A. (2003). - Les théories de la complexitité chez les géographes, Anthropos, París.

De Mattos C., Ducci M.E., Rodriguez A., YANEZ G. (eds.). (2004). - Santiago en la Globalización ¿una nueva ciudad?, Ediciones Sur y EURE libros, Santiago. 
HILLIER B. (1998). - The Common Language of Space: a way of looking at the social, economic an environmental functioning of cities in a common basis, URL : http://www.barlett.cl.ac.uk/ spacesyntax/publications/commolang.html

HILLIER B. \& HANSEN J. (1984). - The social logic of space, Cambridge University Press, Cambridge.

KunZMANN K.K. (2001). - « WelcheZukünfte für Suburbia ? Acht Inseln im Archipel der Stadtregion ». En : Brake, K., Dangschat, J. S. y Herfert, G. (eds.) : Suburbanisierung in Deutschland. Aktuelle Tendenzen, Opladen, 213-221.

Monclus F.J. (ed.), (1998). - La ciudad dispersa, Centre de Cultura Contemporània, Barcelona.

ORTIZ J. \& ARAVENA E. (2002). - « Migraciones intraurbanas y nuevas periferias en Santiago de Chile : efectos en la sociogeografía de la ciudad». Revista GeoFocus N², pp.49.60, Madrid, España. http://geofocus.rediris.es/articulos2002.html

ORTIZ J. \& EsColano S. (2005). - « Crecimiento periférico del Gran Santiago ¿Hacia la desconcentración funcional de la ciudad ? ». En : VII Coloquio Internacional de Geocrítica : Los agentes urbanos y las políticas, Santiago de Chile, 24-27 de mayo de 2005, Instituto de Geografía, Pontificia Universidad Católica. www.ub.es/geocrit/7-col.htm

ORTIZ J. \& MORALES S. (2002). - « Impacto socioespacial de las migraciones intraurbanas en entidades de centro y de nuevas periferias del Gran Santiago », Eure, vol. XXVIII, 85, 171-185.

PORTUGAli J. (2000). - Self-organization and Cities, Springer-Verlag, Heidelberg.

READ S \& BUDIARTO L. (2003). - « Human scales : understanding places of centering and decentering ». En : Procedings, 4rd. International Space Sintax Symposium, Londres, 13.1-13.16.

SASSEN S. (1991). - The Global City : New York, London, Tokyo, Princeton Univ. Press, Princeton traducción al español : EUDEBA, 1997, Buenos Aires).

WILSON A.G. (2000). - Complex spatial systems : the modelling foundations of urban and regional analysis, Pearson Education, London.

\section{NOTES}

1. Proyectes FONDECYT nos : 7040151 et 1040707.

2. La "syntaxe de l'espace " a développé un remarquable corpus propre de concepts et de techniques d'analyse. Le principal se trouve dans l'œuvre de Hillier et Hanson (1984), qui a été considéré comme la bible de cette théorie. D’autres apports conceptuels, méthodologiques et des applications se trouvent dans les actes des quatre symposia réalisés (http:// www.spacesyntax.com).

3. Une configuration spatiale se définit comme un domaine continu et connecté d'occupation et de mouvement potentiel qui émerge de la disposition de frontières. Cette notion est plus vaste que celle de structure spatiale, car les objets interviennent entre eux à travers des tiers, c'est à dire que les changements locaux altèrent l'ensemble du système. Les procédés d'analyse poursuivent la description des propriétés d'une configuration déterminée pour les associer à d'autres variables représentatives d'autres phénomènes.

4. La topologie d'un réseau, comme celui de rues, est représentée au moyen de graphes (G) composés de sommets (nœuds, intersections), et des arêtes qui sont les segments qui connectent les nœuds. L'ordre ou la hiérarchie de chaque nœud peut s'exprimer par le nombre de segments qui le connectent à d'autres nœuds. 
5. Un espace constitué est un " espace convexe » (surface définie par l'ensemble de points visibles entre eux) délimité par des bâtiments qui ont une entrée directe depuis celui-ci ; un espace non constitué, par contre, n'a pas de porte d'accès direct aux bâtiments.

6. La profondeur topologique s'exprime par le nombre de niveaux (contrôles de passage) nécessaires pour y arriver depuis une zone de transit (une rue).

7. Dans la «syntaxe de l'espace " l'intégration globale (RN) est une mesure d'accessibilité d'une ligne axiale (une rue) qui mesure la fréquence avec laquelle une ligne est utilisée pour faire un parcours complet entre toutes les lignes du système par l'itinéraire le plus court (plus petit nombre de virages).

8. Les catégories d'utilisation du sol sont les suivantes : résidentielle, industrielle, commerciale, bureaux, administration publique et défense, santé, culte, instruction, hôtel et motel, stationnement, transport, stockage et cave, espaces verts, mines, autres...

9. Les activités du sol non résidentiel sont réalisées par des entreprises (ou institutions) qui nécessitent et exigent un effort pour leur maintien et sont aussi des pôles d'attractions de flux de personnes et de biens. Par conséquent, ce sont les éléments principaux générateurs de diversité et de complexité.

\section{RÉSUMÉS}

Dans cet article sont étudiées quelques caractéristiques de la fragmentation des structures spatiales du Grand Santiago (Chili) dans le contexte de son expansion récente. En général, quelques structures spatiales, comme celle du tissu construit ou celle des utilisations du sol, montrent des processus de fragmentation au moins dans deux directions: d'une part, la discontinuité augmente parce que les éléments constituants se séparent davantage et, d'autre part, la façon de s'organiser de ceux-ci varie. En ce qui concerne le tissu construit, le changement de l'organisation est une propriété émergente de l'utilisation massive des grandes unités d'occupation de l'espace. Il est probable que les processus de fragmentation aient une relation entre eux, et exercent une certaine influence sur la croissance de la ségrégation spatiale et sur la conformation d'un modèle de ville physique et fonctionnel de genre granulaire, postsuburbia, dont le Grand Santiago montre déjà quelques traits.

In this article we examine some characteristics of the fragmentation of spatial structures in Greater Santiago (Chile) in the context of its recent expansion. Some spatial structures, such as those of urban morphology or land use, reveal processes of fragmentation in at least two ways. On the one hand, discontinuity grows because constituent elements become more separated; on the other hand, the way that these elements are organized changes. In terms of urban morphology, change in organization emerges from the use of large units that occupy space. It is probable that the processes of fragmentation are inter-related, and influence the growth of spatial segregation and the construction of a model of a physical and functional city that is granular and post-suburban. Greater Santiago demonstrates some of these features.

Die Metropolen Lateinamerikas wachsen heute nur noch wenig hinsichtlich ihrer Einwohnerzahlen, wohl aber in der Fläche. Dabei wandeln sich die stadtmorphologischen Strukturen ebenso wie jene der Landnutzung. Diese Entwicklungen können als Fragmentierungsprozesse verstanden werden. Dabei wächst die Diskontinuität, weil ehemals 
zusammenhänge Elemente zunehmend separiert werden. Dabei entstehen ganz neue räumliche Organisationsformen. Es scheint, als ob die Fragmentierungsprozesse in Wechselbeziehungen stehen und dabei sozialräumliche Segregationsprozesse stimulieren. Insgesamt entsteht ein quasi gekörnter, granularer Stadtrorganismus, der in außerhalb des Kernraums post-suburbane Strukturen aufweist. In Groß-Santiago können einige dieser Strukturen klar demonstriert werden.

\section{INDEX}

Keywords : complexity, fragmentation, Gran Santiago, postsuburbia, spatial structures Mots-clés : complexité, fragmentation, Grand Santiago, postsuburbia, structures spatiales Schlüsselwörter : Fragmentierung, Groß-Santiago, Komplexität, Postsuburbia, räumliche Strukturen

\section{AUTEURS}

\section{SEVERINO ESCOLANO UTRILLA}

Service de Géographie et Aménagement du Territoire, Université de Saragosse (Espagne), severino@unizar.es

\section{JORGE ORTIZ VELIZ}

Service de Géographie Faculté d'Architecture et Urbanisme, Université de Santiago (Chili), jortiz@abello.dic.uchile.cl 